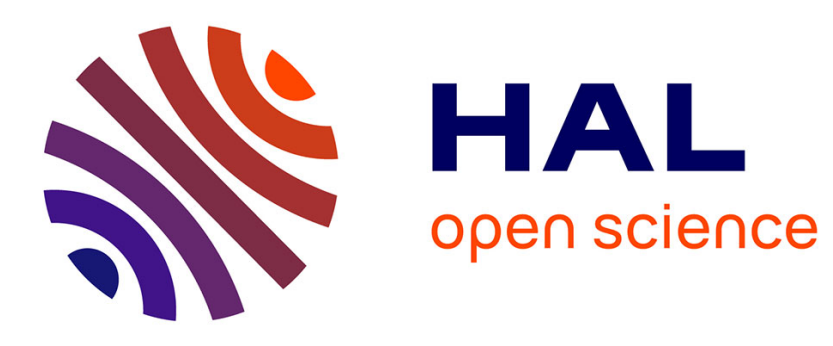

\title{
Observer design for nonlinear systems described by multiple models
}

Rodolfo Orjuela, Benoît Marx, José Ragot, Didier Maquin

\section{To cite this version:}

Rodolfo Orjuela, Benoît Marx, José Ragot, Didier Maquin. Observer design for nonlinear systems described by multiple models. 7th IFAC Symposium on Fault Detection, Supervision and Safety of Technical Processes, Safeprocess 2009, Jun 2009, Barcelona, Spain. pp.CDROM, 10.3182/200906304-ES-2003.00003 . hal-00374959

\section{HAL Id: hal-00374959 \\ https://hal.science/hal-00374959}

Submitted on 25 Mar 2014

HAL is a multi-disciplinary open access archive for the deposit and dissemination of scientific research documents, whether they are published or not. The documents may come from teaching and research institutions in France or abroad, or from public or private research centers.
L'archive ouverte pluridisciplinaire HAL, est destinée au dépôt et à la diffusion de documents scientifiques de niveau recherche, publiés ou non, émanant des établissements d'enseignement et de recherche français ou étrangers, des laboratoires publics ou privés. 


\title{
Observer design for nonlinear systems described by multiple models
}

\author{
Rodolfo Orjuela, Benoît Marx, José Ragot and Didier Maquin \\ Centre de Recherche en Automatique de Nancy \\ UMR 7039, Nancy-Université, CNRS \\ 2, Avenue de la Forêt de Haye, F 54516 Vandouvre-lès-Nancy, France \\ $\{$ Rodolfo.Orjuela, Benoit.Marx, Jose.Ragot, \\ Didier.Maquin\}@ensem.inpl-nancy.fr
}

\begin{abstract}
This paper addresses both analysis and observer design for nonlinear systems modelled by decoupled multiple models. With respect to classic used multiple models, the decoupled multiple model is characterized by heterogeneous submodels in the sense that their state spaces may be of various dimensions. Thanks to this fact, flexibility and generality is introduced in the modelling stage. The main contribution of the paper is the development of new sufficient conditions on LMI form for ensuring the exponential convergence towards zero of the estimation error in the continuous and in the discrete-time. The new proposed conditions enable to obtain a better decay rate with respect to the existing conditions. The validity of the proposed methodology and its application to sensor faults detection and isolation is illustrated by an academic example.
\end{abstract}

Keywords: state estimation; nonlinear systems; sensor faults; multiple models; decoupled multiple model; heterogeneous local model networks

\section{INTRODUCTION}

Context. Nowadays, fault diagnosis systems is becoming unavoidable in order to ensure the safety of systems and operators. In order to setting up a fault diagnosis system strategy, sensitive signals to faults must be available. These signals can be obtained, for example, with the help of state estimation techniques [Patton et al., 2000],[Isermann, 2006]. Indeed, the state estimation of a system provided by an observer, is an intermediary stage to estimate the characteristic variables of the system such as its outputs. These estimations can be considered as an analytical source of redundancy of the system. With the help of this source of redundancy, fault diagnosis tasks (detection, isolation and identification) can be effectively accomplished by analysing the signals sensitive to faults (residual signals) generated by the discrepancies between the measured outputs of the system and their estimated. Indeed, if the residual signal deviates from zero then an abnormal behaviour of the system can be detected. Consequently, the observer design plays an important role in the described fault diagnosis strategy.

Note however that the state estimation problem (i.e. observer design) can be particularly arduous when the dynamic behaviour of the system must be represented by nonlinear models. A nonlinear representation of the system is generally needed when the whole operating space of the system must be considered (global modelling) instead of a reduced operating space for example around an operating point (local modelling). This situation frequently arise in a fault diagnosis strategy because all admissible dynamic behaviours of the system must be taken into consideration. In order to capture highly nonlinear dynamic behaviours, in a wide operational range of the system, nonlinear representations based on local model networks also known as multiple model approach can be used as an appropriate global modelling tool [Murray-Smith and Johansen, 1997]. On the other hand, by means of this representation the specific analysis of the non-linearity of the system can be avoided and the observer design can be in this way simplified.

Tools and proposed method. In the multiple model approach the use of an only one complex model is avoided by introducing a set of submodels correctly interconnected by means of an interpolation mechanism. The multiple models are able to accurately capture the nonlinear behaviour of a large class of systems in the whole operating space. In this modelling strategy, the operating space of the system under investigation is decomposed in a finite number of operating zones. The dynamic behaviour of the system, inside each operating zone, is then modelled by a local model ( submodel), often a linear model of reduced dimension. Finally, according to the current operating point of the system, the contribution of each submodel is more or less taken into consideration thanks to weighting functions.

In this modelling approach, two main structures of multiple models can be considered [Filev, 1991]. In the first one, the submodels have the same state-space and consequently the multiple model is composed of homogeneous submodels. The well known Takagi-Sugeno model proposed, in a fuzzy modelling framework, by Takagi and Sugeno [1985] and in a multiple model modelling framework by Johansen and Foss [1993] is a good example of this kind of multiple model. In the second structure, the multiple model uses heterogeneous submodels, in the sense that the submodels do not share the same state-space. This second structure, so-called decoupled multiple model, will be employed in the present work.

Related work. The main interest for employing heterogeneous submodels lies in the fact that the order (i.e. the state space dimension) of each submodel can be different. Consequently, 
the degree of generality of the decoupled multiple model is undoubtedly increased because a non uniform decomposition of the operating space can be made, i.e. the complexity of the system in each operating zone may be different. Nonlinear systems identification using this multiple model is presented in Venkat et al., 2003, Vinsonneau et al., 2005, Orjuela et al., 2006 and the design of control laws in Gawthrop, 1995, Gatzke and Doyle III, 1999, Gregorcic and Lightbody, 2000, GarcíaNieto et al., 2008. Note however that the state estimation problem of nonlinear systems using this multiple model has been poorly considered in the literature. To the best of the authors' knowledge, only in the few recent works [Uppal et al., 2003, 2006] this structure of multiple is employed in a fault detection and isolation scheme for nonlinear dynamic systems (in these works, the proposed structure is namely Neuro-Fuzzy Decoupling Fault Detection Scheme NFDFDS). However, only a note of the convergence estimation error is proposed in these works and no analytic proof of the convergence of the estimation error is given.

Contribution. This paper refers to the development of new conditions for ensuring exponential convergence towards zero of the estimation error as a further development of previous works [Orjuela et al., 2007, 2008]. With respect to these works, the proposed conditions makes it possible to improve the dynamic performances of the observer. In particular, the convergence velocity of the estimation error can be improved because a better decay rate can be obtained. The analytic proof of the estimation error convergence is proposed using the Lyapunov theory and the existence condition of the observer, in both continuous and discrete-time, are formulated using linear matrix inequalities (LMIs) [Boyd et al., 1994].

Paper organisation. The decoupled multiple model is presented in section 2 . In section 3, sufficient conditions for observer design are remember and new conditions for ensuring the exponential convergence of the estimation error are proposed. Finally, in section 4, the validity of the proposed methodology and its application to sensor faults detection and isolation is illustrated by an academic example.

Notations. $P>0(P<0)$ denotes a positive (negative) definite matrix $P ; X^{T}$ denotes the transpose of matrix $X$ and $\mathrm{I}$ is the identity matrix of appropriate dimension and $\operatorname{diag}\{\}$ is a block diagonal matrix of appropriate dimension.

\section{ON THE DECOUPLED MULTIPLE MODEL}

Modelling complex system by a rigorous mathematical model can be very arduous and even if this model exist its systematic exploitation can be delicate because this model is generally nonlinear. Multiple models offer an excellent way to avoid partially these difficulties.

Definition 1. (Operator $\delta$ ). The operator $\delta$ is the time derivative for continuous-time models, i.e. $\delta(x(t))=\dot{x}(t)$, and the shift operator for discrete-time models, i.e. $\delta(x(t))=x(t+1)$.

The structure of the decoupled multiple model used in the sequel is given by [Orjuela et al., 2008]:

$$
\begin{aligned}
\delta\left(x_{i}(t)\right) & =A_{i} x_{i}(t)+B_{i} u(t), \\
y_{i}(t) & =C_{i} x_{i}(t), \\
y(t) & =\sum_{i=1}^{L} \mu_{i}(\xi(t)) y_{i}(t),
\end{aligned}
$$

where $x_{i} \in \mathbb{R}^{n_{i}}$ and $y_{i} \in \mathbb{R}^{p}$ are respectively the state vector and the output of the $i^{\text {th }}$ submodel; $y \in \mathbb{R}^{p}$ is the output of the multiple model. The matrices $A_{i} \in \mathbb{R}^{n_{i} \times n_{i}}, B_{i} \in \mathbb{R}^{n_{i} \times m}$ and $C_{i} \in \mathbb{R}^{p \times n_{i}}$ are known and constant. Note that the dimensions $n_{i}$ of each state vector $x_{i}$ can be different. Indeed, the submodel contribution is taken into account via a weighted sum of the submodel outputs and consequently the submodels do not share the same state space.

The weighting functions $\mu_{i}(\xi(t))$ are associated to each operating zone. They satisfy the following convex sum properties:

$$
\sum_{i=1}^{L} \mu_{i}(\xi(t))=1 \text { and } 0 \leq \mu_{i}(\xi(t)) \leq 1, \forall i=1 \ldots L, \forall t .
$$

The weighting functions quantify the relative contribution of each submodel to the global model according to the current operating point of the system. The current operating point is taken into account by means of the so-called decision variable $\xi(t)$ which is a characteristic variable of the system (e.g. inputs and/or measured variables). This variable is considered as an accessible signal free of faults. For simplicity, in the sequel, we shall simply write $\mu_{i}(\xi(t))=\mu_{i}(t)$.

Comments on the multiple model representation. The main feature of this multiple model is that the state space dimension of submodels can be different and thanks to this fact, complex systems with variable structure in the operating range can be well modelled. Indeed, the complexity of each submodel can be well adapted to the complexity of the nonlinear system in each operating zone. Thanks to this fact, the number of parameters needed for modelling the system can be reduced and the well known problem of curse of dimensionality of the model can be reduced in a good way. From a practical point of view, matrices $A_{i}, B_{i}$ and $C_{i}$ describe the local behaviour of the system inside of the $i$ th operating zone. Intuitively, the number of submodels is given by the granularity of the operating space decomposition. Multiple model parameters can be obtained by using the direct linearisation of an a priori nonlinear model around some operating points, or alternatively by using an identification procedure as proposed in Venkat et al., 2003, Vinsonneau et al., 2005, Orjuela et al., 2006.

\section{OBSERVER DESIGN}

In this section the observer design based on decoupled multiple model is investigated. New convergence conditions of the estimation error are established based on our previous works [Orjuela et al., 2007, 2008].

\subsection{Preliminaries}

For the simplicity of manipulations, the decoupled multiple model (1) is rewritten in the following compact form:

$$
\begin{aligned}
\delta(x(t)) & =\tilde{A} x(t)+\tilde{B} u(t), \\
y(t) & =\tilde{C}(t) x(t),
\end{aligned}
$$

where:

$$
\begin{aligned}
x(t) & =\left[x_{1}^{T}(t) \cdots x_{i}^{T}(t) \cdots x_{L}^{T}(t)\right]^{T} \in \mathbb{R}^{n}, n=\sum_{i=1}^{L} n_{i}, \\
\tilde{A} & =\operatorname{diag}\left\{A_{1} \cdots A_{i} \cdots A_{L}\right\}, \\
\tilde{B} & =\left[B_{1}{ }^{T} \cdots B_{i}{ }^{T} \cdots B_{L}{ }^{T}\right]^{T}, \\
\tilde{C}(t) & =\left[\mu_{1}(t) C_{1} \cdots \mu_{i}(t) C_{i} \cdots \mu_{L}(t) C_{L}\right] .
\end{aligned}
$$


Remark 1. The matrix $\tilde{C}(t)$ can be rewritten as a weighted sum of matrices as follows:

$$
\tilde{C}(t)=\sum_{i=1}^{L} \mu_{i}(t) \tilde{C}_{i},
$$

where $\tilde{C}_{i}$ is a constant block matrix given by:

$$
\tilde{C}_{i}=\left[\begin{array}{lllll}
0 & \cdots & C_{i} & \cdots & 0
\end{array}\right]
$$

such that the term $C_{i}$ is found on the $i^{\text {th }}$ block column of $\tilde{C}_{i}$.

\subsection{Observer structure}

The proposed observer based on the decoupled multiple model has the following form:

$$
\begin{aligned}
\delta(\hat{x}(t)) & =\tilde{A} \hat{x}(t)+\tilde{B} u(t)-\tilde{K}(y(t)-\hat{y}(t)), \\
\hat{y}(t) & =\tilde{C}(t) \hat{x}(t),
\end{aligned}
$$

where $\hat{x}(t)$ is the state estimation and $\hat{y}(t)$ the output estimation and $\tilde{K} \in \mathbb{R}^{n \times p}$ is the observer gain to be determined such as the exponential convergence of $\hat{x}(t)$ towards $x(t)$ is guaranteed. Note that the exponential convergence is investigated in order to introduce dynamic performances of the observer.

Remark 2. The outputs $y_{i}(t)$ of the submodels are "artificial modelling signals" only used in order to provide an approximation of the output of the real system. Therefore the outputs $y_{i}(t)$ cannot be employed as accessible signals for driving an observer.

\subsection{Convergence condition of the estimation error}

Let us define the state estimation error:

$$
e(t)=x(t)-\hat{x}(t) \text {. }
$$

The estimation error dynamics is obtained by applying the operator $\delta$ to (8), and by using the equations (3) and (7):

$$
\delta(e(t))=A_{o b s}(t) e(t),
$$

where $A_{o b s}(t)$ is given by:

$$
\begin{aligned}
A_{o b s}(t) & =\sum_{i=1}^{L} \mu_{i}(t) \phi_{i}, \\
\phi_{i} & =\tilde{A}+\tilde{K} \tilde{C}_{i} .
\end{aligned}
$$

Remark 3. The observer design based on each submodel cannot guarantee in a general way the convergence towards zero of the estimation error. Indeed, the matrix $A_{o b s}(t)$ is a timevarying matrix and consequently the combination of the submodel contributions must be taken into consideration in the observer design stage. The observer gain $\tilde{K}$ must be selected in order to ensure the convergence of the estimation error for an arbitrary combination of the submodel contributions and for any initial conditions. For this purpose, the Lyapunov method is employed.

Theorem 1. (Continuous-time case). Consider the decoupled multiple model (3) and the observer (7). The exponential convergence of the estimation error (8) is guaranteed if there exists a symmetric and positive definite matrix $P$, a matrix $G$ and a positive scalar $\alpha$ such that:

$$
(\tilde{A}+\alpha \mathrm{I})^{T} P+P(\tilde{A}+\alpha \mathrm{I})+\left(G \tilde{C}_{i}\right)^{T}+G \tilde{C}_{i}<0, i=1 \ldots L(12)
$$

where $\alpha$ is the decay rate. The observer gain is given by $\tilde{K}=P^{-1} G$.

Proof. See [Orjuela et al., 2008].

Theorem 2. (Discrete-time case). Consider the decoupled multiple model (3) and the observer (7). The exponential convergence of the estimation error (8) is guaranteed if there exists a symmetric and positive definite matrix $P$, a matrix $G$ and a positive scalar $\alpha$ such that:

$$
\left[\begin{array}{cc}
(1-2 \alpha) P & \tilde{A}^{T} P+\tilde{C}_{i}^{T} G^{T} \\
P \tilde{A}+G \tilde{C}_{i} & P
\end{array}\right]>0, \quad i=1 \ldots L
$$

where $\alpha$ is the decay rate. The observer gain is given by $\tilde{K}=P^{-1} G$.

Proof. See [Orjuela et al., 2007].

Comments and discussion on the observability issues. The augmented matrices $\tilde{A}$ and $\tilde{C}_{i}$ have been introduced in order to establish the observer existence conditions of the observer. The exponential convergence of the estimation error is carried out by considering a decay rate $\alpha$ different to zero. Note however that the choice of this decay rate can be limited by the particular form of matrices $\tilde{A}$ and $\tilde{C}_{i}$ used in theorems 1 and 2 . Indeed, all columns of matrix $\tilde{C}_{i}$ are equal to zero except the $i^{\text {th }}$ block column. Consequently, eigenvalues assignment of matrix $A_{\text {obs }}(t)$ may be limited because de pair $\left(\tilde{A}, \tilde{C}_{i}\right)$ is not observable.

In order to partially avoid this problem new convergence conditions are established by introducing new augmented matrices.

\subsection{New convergence conditions}

In this section the main contribution of the paper are presented. New convergence conditions of the estimation error are proposed by considering the matrix $A_{o b s}(t)$, in (9), as constant matrix with some "artificial" norm-bounded uncertainties due to the weighting functions. For this purpose, the mean value of the matrices $\tilde{C}_{i}$ is introduced:

$$
\tilde{C}_{0}=\frac{1}{L} \sum_{i=1}^{L} \tilde{C}_{i}=\frac{1}{L}\left[\begin{array}{llll}
C_{1} & C_{2} & \cdots & C_{L}
\end{array}\right] .
$$

By taking into consideration

$$
\begin{aligned}
\tilde{C}_{i} & =\tilde{C}_{i}+\tilde{C}_{0}-\tilde{C}_{0}, \\
& =\bar{C}_{i}+\tilde{C}_{0},
\end{aligned}
$$

the matrix $A_{o b s}(t)$, defined by (10), becomes

$$
\begin{aligned}
A_{o b s}(t) & =\tilde{A}+\sum_{i=1}^{L} \mu_{i}(t) \tilde{K} \tilde{C}_{i}, \\
& =\tilde{A}+\tilde{K} \tilde{C}_{0}+\sum_{i=1}^{L} \mu_{i}(t) \tilde{K} \bar{C}_{i}
\end{aligned}
$$

where $\bar{C}_{i}=\tilde{C}_{i}-\tilde{C}_{0}$. Now, the matrix $A_{o b s}(t)$ can then considered as constant matrix with norm-bounded uncertainties due to the weighting functions.

Lemma 1. For any constant real matrices $X$ and $Y$ with appropriate dimensions, a matrix function $F(t)$ bounded-norm, 
i.e. $F^{T}(t) F(t) \leq \mathrm{I}$, then the following property holds for any positive matrix $Q$

$$
X F(t) Y+Y^{T} F^{T}(t) X^{T} \leq X Q^{-1} X^{T}+Y^{T} Q Y .
$$

Lemma 2. (Schur complement). The following two inequalities are equivalent:

(1) $\left[\begin{array}{cc}Q & S \\ S^{T} & R\end{array}\right]>0$ where $Q=Q^{T}$ and $R=R^{T}$

(2) $R>0, Q-S R^{-1} S^{T}>0$.

The convergence towards zero of the estimation error can be investigated as previously by considering the following candidate quadratic Lyapunov function:

$$
V(t)=e^{T}(t) P e(t), \quad P>0 \quad P=P^{T} .
$$

\section{Continuous-time case}

Theorem 3. (Continuous-time case). Consider the decoupled multiple model (3) and the observer (7). The exponential convergence towards zero of the estimation error (8) is guaranteed if there exists symmetric and positive definite matrices $P$ and $Q$, a matrix $G$ and a positive scalar $\alpha$ such that:

$$
\left[\begin{array}{ccc}
\Delta & \bar{G} & \overline{\bar{C}}^{T} Q \\
\bar{G}^{T} & -Q & 0 \\
Q \overline{\bar{C}} & 0 & -Q
\end{array}\right]<0
$$

where

$$
\begin{aligned}
\Delta & =P(\tilde{A}+\alpha \mathrm{I})+(\tilde{A}+\alpha \mathrm{I})^{T} P+G \tilde{C}_{0}+\left(G \tilde{C}_{0}\right)^{T}, \\
\tilde{C}_{0} & =\frac{1}{L} \sum_{i=1}^{L} \tilde{C}_{i}, \\
\bar{G} & =[G \cdots G \cdots G], \\
\overline{\bar{C}} & =\left[\bar{C}_{1}^{T} \cdots \bar{C}_{i}^{T} \cdots \bar{C}_{L}^{T}\right]^{T}, \\
\bar{C}_{i} & =\tilde{C}_{i}-\tilde{C}_{0} .
\end{aligned}
$$

where $\alpha$ is the decay rate and the observer gain is given by $\tilde{K}=P^{-1} G$.

Proof. The exponential convergence towards zero of the estimation error is guaranteed if the following inequality is ensured [Boyd et al., 1994]

$$
\exists \alpha>0: \dot{V}(t)<-2 \alpha V(t),
$$

where $\alpha$ is the so called decay rate.

By using the Lyapunov function (19) and the definition of $\delta(e(t))$, given by (9), then the inequality (21) is ensured if the following inequality holds:

$$
P A_{o b s}(t)+A_{o b s}^{T}(t) P+2 \alpha P<0 .
$$

Considering the new definition (18) of $A_{o b s}(t)$ then the inequality (22) becomes

$$
\begin{aligned}
P \tilde{A} & +G \tilde{C}_{0}+\tilde{A}^{T} P+\left(G \tilde{C}_{0}\right)^{T}+2 \alpha P \\
& +\sum_{i=1}^{L} \mu_{i}(t) G \bar{C}_{i}+\sum_{i=1}^{L} \mu_{i}(t)\left(G \bar{C}_{i}\right)^{T}<0,
\end{aligned}
$$

where $G=P \tilde{K}$. The previous inequality can be rewritten as follows:

$$
\begin{aligned}
P \tilde{A}+\tilde{A}^{T} P & +G \tilde{C}_{0}+\left(G \tilde{C}_{0}\right)^{T}+2 \alpha P \\
& +\bar{G} F(t) \overline{\bar{C}}+(\bar{G} F(t) \overline{\bar{C}})^{T}<0,
\end{aligned}
$$

where

$$
\begin{aligned}
\bar{G} & =[G \cdots G \cdots G], \\
F(t) & =\operatorname{diag}\left\{\mu_{1}(t) \mathrm{I} \cdots \mu_{\mathrm{i}}(\mathrm{t}) \mathrm{I} \cdots \mu_{\mathrm{L}}(\mathrm{t}) \mathrm{I}\right\}, \\
\overline{\bar{C}} & =\left[\bar{C}_{1}^{T} \cdots \bar{C}_{i}^{T} \cdots \bar{C}_{L}^{T}\right]^{T} .
\end{aligned}
$$

Let us notice that the matrix $F(t)$ in (24) is norm-bounded (i.e. $F^{T}(t) F(t) \leq \mathrm{I}$ ) because the weighting functions satisfy $0 \leq \mu_{i}(t) \leq 1$.

With the help of lemma 1 then the inequality (24) is guaranteed if the following inequality holds:

$$
\begin{array}{r}
P(\tilde{A}+\alpha \mathrm{I})+(\tilde{\mathrm{A}}+\alpha \mathrm{I})^{\mathrm{T}} \mathrm{P}+\mathrm{G} \tilde{\mathrm{C}}_{0}+\left(\mathrm{G} \tilde{\mathrm{C}}_{0}\right)^{\mathrm{T}} \\
+\bar{G} Q^{-1} \bar{G}^{T}+\overline{\bar{C}}^{T} Q \overline{\bar{C}}<0 .
\end{array}
$$

Note that the previous inequality is not in a LMI form. However, using two times the Schur complement (see lemma 2) the inequality (26) becomes an LMI and this completes the proof of theorem 3 .

\section{Discrete-time case}

Theorem 4. (Discrete-time case). Consider the decoupled multiple model (3) and the observer (7). The exponential convergence towards zero of the estimation error (8) is guaranteed if there exists symmetric and positive definite matrices $P$ and $Q$, a matrix $G$ and a positive scalar $\alpha$ such that:

$$
\left[\begin{array}{ccc}
(1-2 \alpha) P-\overline{\bar{C}}^{T} Q \overline{\bar{C}} & \left(P \tilde{A}+G \tilde{C}_{0}\right)^{T} & 0 \\
P \tilde{A}+G \tilde{C}_{0} & P & \bar{G} \\
0 & \bar{G}^{T} & Q
\end{array}\right]>0
$$

where

$$
\begin{aligned}
\tilde{C}_{0} & =\frac{1}{L} \sum_{i=1}^{L} \tilde{C}_{i}, \\
\bar{G} & =[G \cdots G \cdots G], \\
\overline{\bar{C}} & =\left[\bar{C}_{1}^{T} \cdots \bar{C}_{i}^{T} \cdots \bar{C}_{L}^{T}\right]^{T}, \\
\bar{C}_{i} & =\tilde{C}_{i}-\tilde{C}_{0} .
\end{aligned}
$$

where $\alpha$ is the decay rate and the observer gain is given by $\tilde{K}=P^{-1} G$.

Proof. The exponential convergence towards zero of the estimation error in the discrete-time case is investigated in a similar way. Consider the same candidate quadratic Lyapunov function as (19). The exponential convergence is guaranteed if the following inequality is ensured

$$
\exists \alpha>0: \Delta V(t)<-2 \alpha V(t),
$$

where $\Delta V(t)=V(t+1)-V(t)$.

By using the Lyapunov function (19) and the estimation error dynamics given by (9), this inequality becomes

$$
A_{o b s}^{T}(t) P A_{o b s}(t)-(1-2 \alpha) P<0
$$

By applying the Schur complement the obtained inequality (29) becomes 


$$
\left[\begin{array}{cc}
(1-2 \alpha) P & A_{o b s}^{T}(t) P \\
P A_{o b s}(t) & P
\end{array}\right]>0
$$

Using the expression of $A_{o b s}(t)$ given by (18), this last expression can be rewritten as follows:

$$
\left[\begin{array}{cc}
(1-2 \alpha) P & \Delta^{T} \\
\Delta & P
\end{array}\right]+X F(t) Y+Y^{T} F(t) X^{T}>0
$$

where

$$
\Delta=P \tilde{A}+G \tilde{C}_{0}, G=P \tilde{K}, X=\left[\begin{array}{ll}
0 & \bar{G}^{T}
\end{array}\right]^{T}, Y=\left[\begin{array}{ll}
\overline{\bar{C}} & 0
\end{array}\right] .
$$

and where matrices $\bar{G}, F(t)$ and $\overline{\bar{C}}$ are defined in (25). The proof of theorem 4 is completed by using the lemma 1 and the Schur complement.

Comments and discussion on the observability issues. Notice that in the previous theorems the matrix $\tilde{C}_{i}$ is not directly used for decay rate selection. Indeed, the supplementary matrix $\tilde{C}_{0}$ introduce some degrees of freedom for decay rate choice because the rank of $\tilde{C}_{0}$ is greater than the rank of matrices $\tilde{C}_{i}$ and the provides a better observability of the system. Indeed, the pair $\left(\tilde{A}, \tilde{C}_{0}\right)$ in theorems 3 and 4 are observable.

\section{SIMULATION EXAMPLE}

Consider the continuous-time decoupled multiple model with $L=2$ different dimension submodels given by:

$$
\begin{aligned}
A_{1} & =\left[\begin{array}{ccc}
-2.0 & 0.5 & 0.6 \\
-0.3 & -0.9 & -0.5 \\
-1.0 & 0.6 & -0.8
\end{array}\right], & A_{2} & =\left[\begin{array}{cc}
-0.8 & -0.4 \\
0.1 & -1.0
\end{array}\right], \\
B_{1} & =\left[\begin{array}{lll}
1.0 & 0.8 & 0.5
\end{array}\right]^{T}, & B_{2} & =\left[\begin{array}{ll}
-0.5 & 0.8
\end{array}\right], \\
C_{1} & =\left[\begin{array}{ccc}
0.9 & -0.8 & -0.5 \\
-0.4 & 0.6 & 0.7
\end{array}\right], & C_{2} & =\left[\begin{array}{cc}
-0.8 & 0.6 \\
0.4 & -0.7
\end{array}\right] .
\end{aligned}
$$

Here, the decision variable $\xi(t)$ is the input signal $u(t) \in[0,1]$. The weighting functions are obtained from normalised Gaussian functions:

$$
\begin{aligned}
& \mu_{i}(\xi(t))=\eta_{i}(\xi(t)) / \sum_{j=1}^{L} \eta_{j}(\xi(t)), \\
& \eta_{i}(\xi(t))=\exp \left(-\left(\xi(t)-c_{i}\right)^{2} / \sigma^{2}\right),
\end{aligned}
$$

with the $\sigma=0.5, c_{1}=0.25$ and $c_{2}=0.75$.

Conditions of theorem 1 are fulfilled with:

$$
\tilde{K}=\left[\begin{array}{lllll}
-0.546 & -0.119 & -0.142 & -0.078 & 0.057 \\
-0.647 & -0.109 & -0.148 & -0.019 & 0.038
\end{array}\right]^{T}
$$

with a decay rate $\alpha=0.8$. Note that solutions satisfying conditions of theorem 1 are not found for a decay rate $\alpha>0.8$

On the other hand, conditions of theorem 3 are fulfilled with:

$$
\tilde{K}=\left[\begin{array}{ccccc}
0.507 & -0.082 & 0.539 & -1.674 & 1.393 \\
-0.153 & 0.115 & -0.200 & 0.520 & -0.945
\end{array}\right]^{T}
$$

with a decay rate $\alpha=1$. Note that solutions satisfying conditions of theorem 3 are found for a decay rate $\alpha>0.8$.

State estimation errors provided by these two observers are depicted in figures 1 and 2 . Let us notice that the initial conditions of the multiple model are not null and the initial conditions of the observers are null. It can be seen from these figures that the observer design using theorem 3 provides a better decay rate and good dynamics performances of the estimation errors.
However a great decay rate is obtained by a high gain and this fact is at the origin of undesirable phenomenons, for example an amplification of the noise. The choice of the observer gain is subject to a trade-off between the noise amplification and the decay rate.

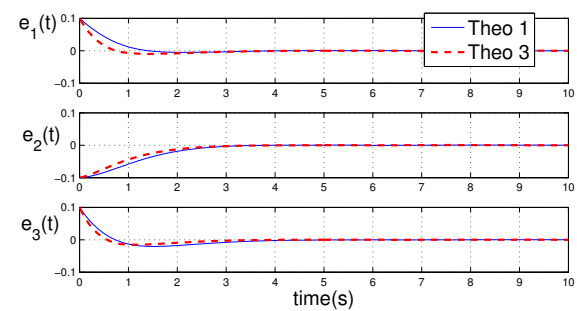

Figure 1. State estimation errors of submodel 1

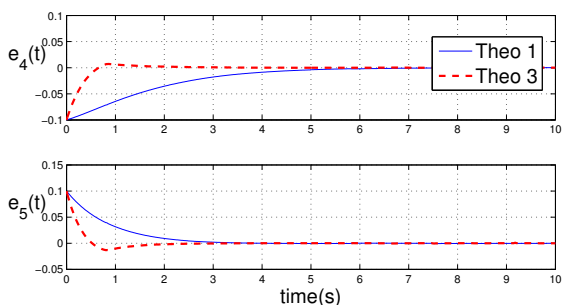

Figure 2. State estimation errors of submodel 2

\section{Application to sensor faults diagnosis}

The state estimation provided by the proposed observer can be used in a classic way for fault diagnosis of nonlinear systems represented by means of a decoupled multiple model, as proposed in Orjuela et al. [2008]. For example, sensor fault detection and isolation task can be well achieved by structuring judiciously the residual signals with the aid of an observer schemes (e.g. Dedicated and/or Generalized Observer Schemes) and by using an appropriated logical decision rule [Patton et al., 2000],[Isermann, 2006].

Here, two dedicated observers are designed by using the new conditions proposed by the theorem 3 . The first dedicated observer is driven only by the first output of the system and the second one by the second output. Each dedicated observer provides an estimation of the system outputs, noted by $\hat{y}_{i}^{o b s}(t)$ where $i$ is the number of the estimated output and obs is the considered observer. Hence, signals sensitive to sensor faults acting on the system, residuals $r_{i, o b s}(t)$, can be easily generated by considering the discrepancies between the measured outputs of the system $y_{i}(t)$ and their estimated $\hat{y}_{i}^{o b s}(t)$. Sensor faults detection and isolation are accomplished by considering the simultaneous configuration of the obtained residual signals $r_{i, o b s}(t)$ (see the fault signatures of different faults).

\begin{tabular}{c|c|c|c|c|}
\cline { 2 - 5 } \multicolumn{1}{c|}{} & $r_{1,1}$ & $r_{2,1}$ & $r_{1,2}$ & $r_{2,2}$ \\
\hline \hline$\delta_{1}$ & $?$ & $?$ & 1 & 0 \\
\hline$\delta_{2}$ & 0 & 1 & $?$ & $?$ \\
\hline
\end{tabular}

Table 1. Fault signatures of different faults

The fault signatures of different faults $\delta_{i}$ is built as follows. Considering that $y_{1}(t)$ is free of fault, if a sensor fault $\delta_{2}$ acts on the second output of the system then the sensitive signals obtained with the help of the first observer are, in theory, $r_{1,1}(t)=0$ and $r_{2,1}(t)=1$. Note that the configuration of the residual signals generated by the second observer $r_{i, 2}(t)$ cannot 
be taken into consideration because this observer is driven by the corrupted output $y_{2}(t)$. Consequently, configuration of $r_{i, 2}(t)$ is represented by "? " in the fault signatures table 1 . When a sensor fault $\delta_{1}$ acts on the first output then the residuals obtained with the help of the second observer are $r_{1,2}(t)=1$ and $r_{2,2}(t)=0$. In the same way, the residual signals $r_{i, 1}(t)$ are not taken into consideration (configuration of $r_{i, 1}(t)$ is represented by "?"). A logical decision rule can be then established on the basis of the proposed configuration of the residuals $r_{i, o b s}(t)$ summarized in table 1 (see [Orjuela et al., 2008] for details).

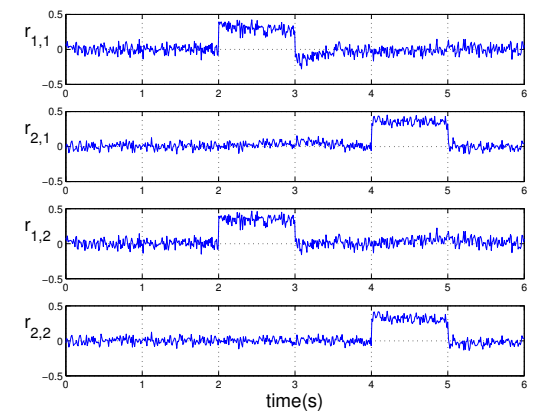

Figure 3. Evolution of the residual signals

In this simple example, two constant sensor faults $\delta_{1}$ and $\delta_{2}$ acting respectively on output 1 and output 2 are considered. In the simulation sensor noise are also added on the system outputs. The time evolution of the residual signals are plotted on figure 3 . Considering the evolution of the residual signals $r_{i, 2}(t)$. In the time-interval $t=2 s$ and $t=3 s$, these residual signals are $r_{1,2}(t)=1$ and $r_{2,2}(t)=0$. Consequently, a sensor fault acting on $y_{1}(t)$ can be well detected in this time-interval. On the other hand, the residual signals $r_{i, 1}(t)$, in the time-interval $t=4 \mathrm{~s}$ and $t=5 s$, are $r_{1,1}(t)=0$ and $r_{2,1}(t)=1$. Consequently, a sensor fault acting on $y_{2}(t)$ can be well detected in this time-interval.

\section{CONCLUSIONS}

This paper has proposed a new way for designing a proportional observer based on a decoupled multiple model. Systematic procedure, based on the LMI framework, has been established in order to design an observer which ensures the exponential convergence of the estimation error. The new proposed conditions allow to obtain a better decay rate with respect to the existing conditions. The effectiveness of the proposed approach is illustrated via a simulation example. The application of the proposed observer in a sensor fault detection and isolation is also illustrated.

The feasibility of the new proposed conditions with respect to the previous ones must be investigated in the future in order to stablish the conservatism of the proposed conditions with respect to the previous ones. The use of the proposed approach in a FDI strategy must be investigated in detail in order to ensure, for example, an attenuation level with respect to disturbances and a maximal amplification level with respect to faults.

\section{REFERENCES}

S. Boyd, L. El Ghaoui, E. Feron, and V. Balakrishnan. Linear Matrix Inequalities in System and Control Theory. SIAM studies in applied mathematics. SIAM, Philadelphia, P.A., 1994.
D. Filev. Fuzzy modeling of complex systems. International Journal of Approximate Reasoning, 5(3):281-290, 1991.

S. García-Nieto, M. Martínez, X. Blasco, and J. Sanchis. Nonlinear predictive control based on local model networks for air management in diesel engines. Control Engineering Practice, 16(12):1399-1413, 2008.

E. P. Gatzke and F. J. Doyle III. Multiple model approach for CSTR control. In 14th IFAC World Congress, pages 343348, Beijing, China, 1999.

P.J. Gawthrop. Continuous-time local state local model networks. In 1995 IEEE Conference on Systems, Man and Cybernetics, pages 852-857, Vancouver, British Columbia, 1995.

G. Gregorcic and G. Lightbody. Control of highly nonlinear processes using self-tuning control and multiple/local model approaches. In 2000 IEEE International Conference on Intelligent Engineering Systems, INES 2000, pages 167-171, Portoroz, Slovenia, 2000.

R. Isermann. Fault-Diagnosis Systems. An Introduction from Fault Detection to Fault Tolerance. Springer-Verlag, Berlin Heidelberg, 2006.

T.A. Johansen and B.A. Foss. Constructing NARMAX models using ARMAX model. International Journal Control, 58(5): 1125-1153, 1993.

R. Murray-Smith and T.A. Johansen. Multiple model approaches to modelling and control. Taylor \& Francis, London, 1997.

R. Orjuela, D. Maquin, and J. Ragot. Nonlinear system identification using uncoupled state multiple-model approach. In Workshop on Advanced Control and Diagnosis, ACD'2006, Nancy, France, 2006.

R. Orjuela, B. Marx, D. Maquin, and J. Ragot. State estimation of nonlinear discrete-time systems based on the decoupled multiple model approach. In 4th International Conference on Informatics in Control, Automation and Robotics, ICINCO 2007, Angers, France, 2007.

R. Orjuela, B. Marx, D. Maquin, and J. Ragot. State estimation for nonlinear systems using a decoupled multiple model. International Journal of Modelling Identification and Control, 4(1):59-67, 2008.

R. J. Patton, P.M. Frank, and R.N. Clark. Issues of fault diagnosis for dynamic systems. Springer-Verlag, 2000.

M. Takagi and M. Sugeno. Fuzzy identification of systems and its application to modelling and control. IEEE Transactions on Systems Man and Cybernetics, 15(1):116-132, 1985.

F. J. Uppal, R. J. Patton, and M. Witczak. A hybrid neurofuzzy and de-coupling approach aplied to the DAMADICS benchmark problem. In 5th IFAC Sympsieum on Fault Detection, Supervision and Safety of Technical Processes, SAFEPROCESS, Washington, D.C, USA, 2003.

F. J. Uppal, R. J. Patton, and M. Witczak. A neuro-fuzzy multiple-model observer approach to robust fault diagnosis based on the DAMADICS benchmark problem. Control Engineering Practice, 14(6):699-717, 2006.

A. N. Venkat, P. Vijaysai, and R. D. Gudi. Identification of complex nonlinear processes based on fuzzy decomposition of the steady state space. Journal of Process Control, 13(6): 473-488, 2003.

B. Vinsonneau, D. Goodall, and K. Burnham. Extended global total least square approach to multiple-model identification. In 16th IFAC World Congress, Prague, Czech Republic, 2005. 